\title{
REPRESENTATION OF PLANE ELASTOSTATICS OPERATORS IN DAUBECHIES WAVELETS
}

\author{
S. Dumont and F. Lebon \\ Laboratoire de Mécanique et Génie Civil, Université Montpellier 2. PI. E. Bataillon, \\ 34095 Montpellier Cedex 5, France
}

\begin{abstract}
This paper is devoted to describing representations of the plane elastostatics operators in orthonormal bases of compactly supported wavelets. We are interested in giving an alternative numerical model to the finite element method and Fourier analysis, because wavelets bases are well-suited to adaptivity and discontinuities. The discretization of the elastostatics operators leads to matrices where elementary terms are easily computed from integrals of two or three wavelets and their derivatives. These integrals are performed as eigenvectors of low order matrices and given in filter banks. Mathematical results are worked out in details. A numerical example is presented which demonstrates the efficiency of the representation and shows it being consistent with analytical solutions. Copyright C 1996 Elsevier Science Ltd.
\end{abstract}

\section{INTRODUCTION}

Daubechies [1] has introduced compactly supported wavelets. In Refs [2], [3], Beylkin et al. find exact and explicit representations of several basic operators as derivatives, in orthonormal bases of compactly supported wavelets. In Ref. [4], Beylkin and Torrésani consider the implementation of these operators via filter banks in the framework of the multiresolution analysis. These representations need some regularity concerning the functions transformed by these operators. In elastostatics, displacements are often in Sobolev spaces of low order. We present two examples. lem).

Example 1: periodic homogenization (local prob-

$$
\begin{aligned}
& E \in\left(L^{2}(Y)\right)^{3} \text { be given, find } \mathbf{u} \in\left(H_{\mathrm{p}}^{1}(Y)\right)^{2} \\
& \text { such that } \operatorname{div}^{2} \operatorname{grad}_{\mathrm{s}} \mathbf{u}=-\operatorname{div} \mathrm{CE} .
\end{aligned}
$$

$Y$ is a representative part of a given bidimensional domain $\Omega$ (see Fig. 1). $H_{\mathrm{p}}^{1}(Y)$ is the Sobolev space of first order with periodic conditions on $Y$ (see Section 2). $\mathbf{C}=\mathbf{C}(x)$ is a fourth order tensor depending on the materials.

Example 2: linear elasticity (see Fig. 2 for the notations).

$$
\begin{aligned}
& \mathbf{f} \in\left(L^{2}(\Omega)\right)^{2} \text { and } \mathbf{g} \in\left(L^{2}\left(\hat{\partial}_{\mathrm{L}} \Omega\right)\right)^{2} \text { be given, } \\
& \text { find } \mathbf{u} \in\left(H^{1}(\Omega)\right)^{2} \\
& \text { such that }-\operatorname{div} \operatorname{grad}_{\mathrm{s}} \mathbf{u}=\mathbf{f} \text { in } \Omega \text {, }
\end{aligned}
$$

$$
\operatorname{Cgrad}_{\mathrm{s}} \mathbf{u} \cdot \mathbf{n}=\mathbf{g} \text { on } \hat{o}_{\mathrm{L}} \Omega \text {, }
$$

$$
\mathbf{u}=\mathbf{0} \text { on } \partial_{\mathrm{p}} \Omega \text {. }
$$

This paper is devoted to the representation of plane elastostatics operators [eqns (1) and (2)] in Daubechies bases. This representation is based on a wavelet-Galerkin method. The difficulties arise from the eventual heterogeneities of the materials and to the nature of the differential operator (coupling of the derivatives, two-dimensional operators, second and fourth order tensors, etc).

Mathematical results show that the representation of the elastostatics operators leads to the computation of elementary terms which are integrals of two or three wavelets or scale functions and their derivatives. These wavelets could have different orders (for example Haar basis and Daubechies wavelet of order 3).

These terms are performed via filter banks which are the eigenvectors of matrices or eigenmatrices of a fourth order tensor. Usually, these matrices have a low order and the eigenvectors can be estimated by symbolic computational software.

The paper is organized as follows: in Section 2, generalities concerning bases of compactly supported wavelets are given; Section 3 is devoted to the discretization of the operators using the waveletGalerkin method and to the matrix coefficients computations; Section 4 contains a numerical example to validate the algorithm. Filter banks are given in the Appendices. 


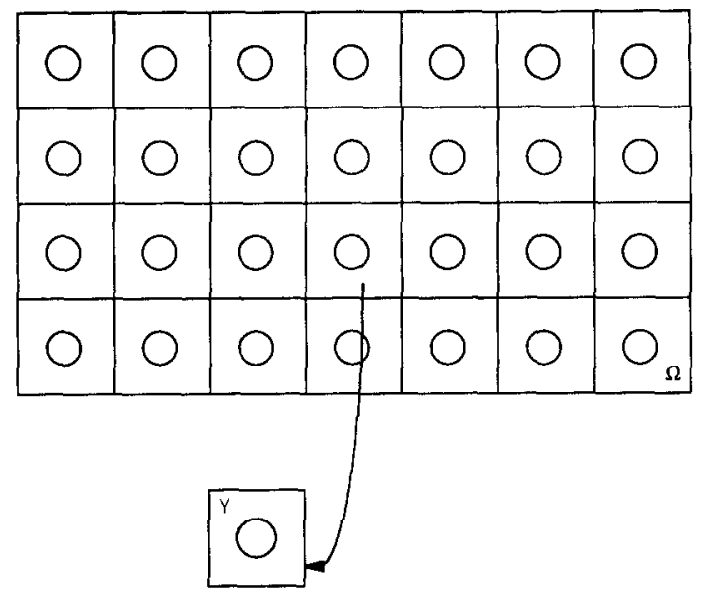

Fig. 1. Heterogeneous bi-dimensional domain and a local representative part.

\section{BASES OF COMPACTLY SUPPORTED WAVELETS}

\subsection{One-dimensional Daubechies wavelets}

In this section, we present a brief summary of one-dimensional Daubechies wavelets [1]. Let $M$ be a positive integer. There exists a sequence of real numbers $\{h(n)\}_{n=0, \ldots, 2 M-1}$ such that

$$
\sum_{n=0}^{n=2 M} h(n)=\sqrt{2},
$$

and

$$
\sum_{n=0}^{n=2 M-1} h(n) h(n+2 k)=\delta_{0 k}, \quad \forall k \in \mathbb{Z},
$$

where $\delta_{i j}=1$ if $i=j, \delta_{i j}=0$ otherwise.

This sequence defines two compactly supported functions $\varphi$ (scale function) and $\psi$ (the associated wavelet). The function $\varphi$ is defined by:

$$
\varphi(x)=\sqrt{2} \sum_{n=0}^{n=2 M-1} h(n) \varphi(2 x-n), \quad \forall x \in \mathbb{R}
$$

and verifies:

$$
\begin{gathered}
\int_{\mathbb{R}} \varphi(x) \mathrm{d} x=1, \\
\int_{\mathbb{R}} \varphi(x) \varphi(x-k) \mathrm{d} x=\delta_{0 k}, \quad \forall k \in \mathbb{Z}, \\
\sum_{k \in \mathbb{Z}} \varphi(x-k)=1, \quad \forall x \in \mathbb{R} .
\end{gathered}
$$

The function $\psi$ is defined by

$$
\psi(x)=\sqrt{2} \sum_{n=0}^{n=2 M-1} g(n) \varphi(2 x-n), \quad \forall x \in \mathbb{R},
$$

where

$$
g(n)=(-1)^{n} h(2 M-n \quad 1), \quad n=0, \ldots, 2 M-1
$$

The compact support of $\varphi$ and $\psi$ is included in the closed interval $[0,2 M-1]$.

The function $\psi$ has $M$ vanishing moments:

$$
\int_{\mathbb{R}} \psi(x) x^{m} \mathrm{~d} x=0 \text { for } m=0, \ldots, M-1,
$$

and verifies

$$
\int_{\mathbb{R}} \psi(x) \psi(x-k) \mathrm{d} x=\delta_{0 k}, \quad \forall k \in \mathbb{Z} .
$$

A representation of the functions $\varphi$ and $\psi$ is given in Fig. 3 for different order of $M$. Let

$$
\varphi_{j k}(x)=2^{j / 2} \varphi\left(2^{j} x-k\right), \quad \forall x \in \mathbb{R}, \quad \forall j, k \in \mathbb{Z},
$$

and

$$
\psi_{j k}(x)=2^{j / 2} \psi\left(2^{j} x-k\right), \quad \forall x \in \mathbb{R}, \quad \forall j, k \in \mathbb{Z} .
$$

The compact support of $\varphi_{j k}$ and $\psi_{j k}$ is included in the closed interval $\left[k / 2^{j},(k+2 M-1) / 2^{j}\right]$.

We define $V_{j}$ as the closure of the space generated by $\left\{\varphi_{j k}, k \in \mathbb{Z}\right\}$ and $W_{j}$, its orthogonal complementary in $V_{j+1}$, as the closure of the space generated by $\left\{\psi_{j k}, k \in \mathbb{Z}\right\}$.

The sub-spaces $V_{j}$ and $W_{i}$ verify the following conditions:

$$
\begin{gathered}
V_{j} \subset V_{j+1}, \\
V_{j+1}=V_{j} \oplus W_{j}, \\
L^{2}(\mathbb{R})=V_{j} \bigoplus_{k \geqslant i} W_{k}=\bigoplus_{k \in \mathbb{Z}} W_{k}, \\
\bigcap_{j \in \mathbb{Z}} V_{j}=\{0\}
\end{gathered}
$$

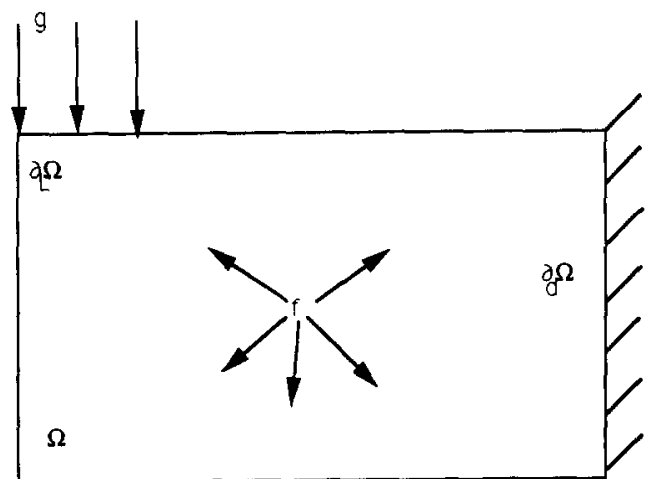

Fig. 2. Two-dimensional elasticity problem. 
(a)
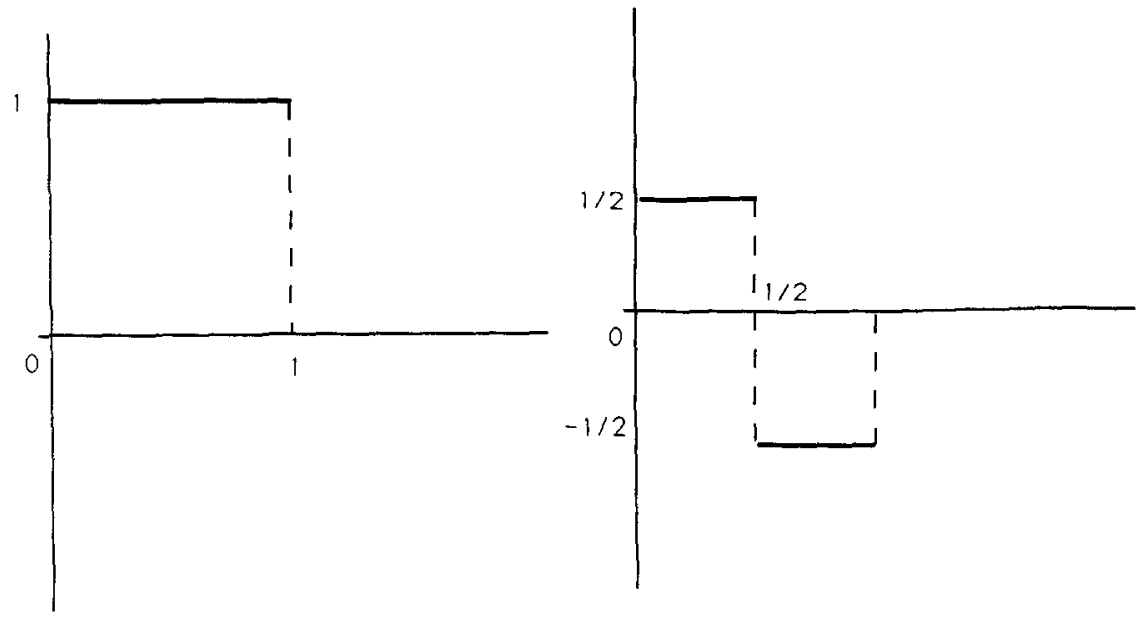

(b)

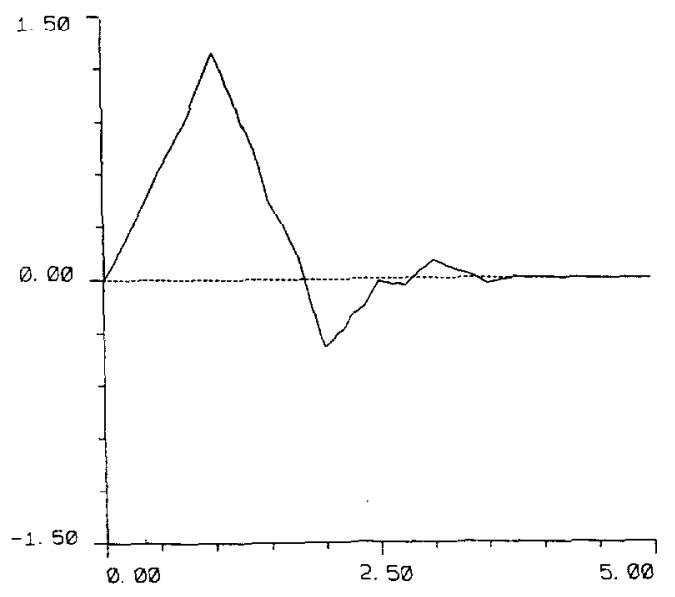

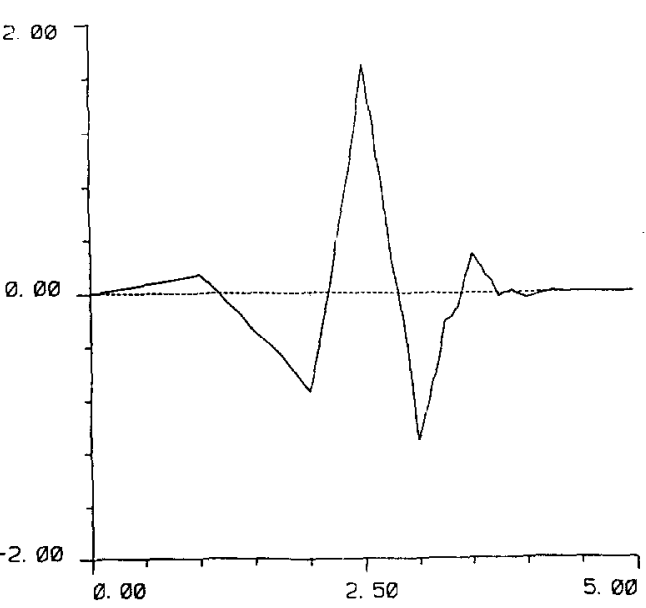

Fig. 3. Scale function and wavelet: (a) $M=1$; (b) $M=3$.

and

$$
L^{2}(\mathbb{R})=\bigcup_{j \in \mathbb{Z}} V_{j}
$$

The set of spaces $V_{j}$ is called a multiresolution analysis of $L^{2}(\mathbb{R})$. These spaces will be used to approximate the solutions of the elastostatics equations using a Galcrkin method. The functions $\varphi$ and $\psi$ give us bases of $L^{2}(\mathbb{R})$ and, for certain values of $M$, bases of $H^{1}(\mathbb{R})$.

Note that the polynomials of degree lower than $M-1$ are exactly included in the space $V_{j}$. For example, for numerical applications we usually use wavelets of order 3 , and so, polynomials of degree 2 are included in the discretization spaces.

\subsection{Periodic one-dimensional wavelets}

It is easy to define periodic wavelets (sce Example 1) and a multiresolution analysis of $L_{\mathrm{p}}^{2}(Y)$. First, we define $L_{\mathrm{p}}^{2}(0,1)$ and $H_{\mathrm{p}}^{1}(0,1)$ by:

$$
L_{\mathrm{p}}^{2}(0,1)=\left\{v \in L_{\mathrm{loc}}^{2}(\mathbb{R}), v(x)=v(x+1) \text { a.e. }\right\}
$$

and

$$
H_{\mathrm{p}}^{\mathrm{l}}(0,1)=\left\{v \in L_{\mathrm{p}}^{2}(0,1), \frac{\partial v}{\partial x_{\mathrm{I}}} \in L^{2}(0,1)\right\}
$$

We define the function $\phi_{j k}$ such that

$$
\phi_{j k}(x)=2^{i / 2} \sum_{r \in \mathbb{Z}} \varphi\left(2^{i}(x+r)-k\right)
$$

One can show [5] that the closure of $\left\{\phi_{j k}, k \in \mathbb{Z}\right\}$, noted $V_{j}^{p}$, defines a multiresolution analysis of $L_{\mathrm{p}}^{2}(0,1)$.

\subsection{Periodic two-dimensional wavelets}

If $Y$ is the square $[0,1]^{2}$, we define $L_{p}^{2}(Y)$ and $H_{\mathrm{p}}^{l}(Y)$ by

$$
\begin{aligned}
L_{\mathrm{p}}^{2}(Y) & =\left\{v \in L_{\mathrm{loc}}^{2}(Y), v\left(x_{1}, x_{2}\right)\right. \\
& =v\left(x_{1}+1, x_{2}\right), v\left(x_{1}, x_{2}\right) \\
& \left.=v\left(x_{1}, x_{2}+1\right) \text { a.e. }\right\}
\end{aligned}
$$


and

$$
H_{\mathrm{p}}^{1}(Y)=\left\{v \in L_{\mathrm{p}}^{2}(Y), \frac{\partial v}{\partial x_{1}} \in L^{2}(Y), \frac{\partial v}{\partial x_{2}} \in L^{2}(Y)\right\}
$$

Furthermore, we can determine the orthonormal basis of $L_{\mathrm{p}}^{2}(Y) . L_{\mathrm{p}}^{2}(Y)=\cup_{j} \mathbb{V}_{j}^{\mathrm{p}}$ where $\mathbb{V}_{j}^{\mathrm{p}}$ is the closure of the space generated by $\left\{\Phi_{k_{1} k_{2}}^{j}, k_{1}, k_{2} \in \mathbb{Z}\right\}$, where

$$
\Phi_{k_{1} k_{2}}^{j}\left(x_{1}, x_{2}\right)=\phi_{j k_{1}}\left(x_{1}\right) \phi_{j k_{2}}\left(x_{2}\right)
$$

It is clear that $\left\{\Phi_{k_{1} k_{2}}^{j}, k_{1}, k_{2} \in \mathbb{Z}\right\}$ is an orthonormal basis of $\mathbb{V}_{j}^{\mathrm{p}}$. With these notations, we obtain $\left(L_{\mathrm{p}}^{2}(Y)\right)^{2}=\left(U_{j} \mathbb{V}_{j}^{\mathrm{p}}\right)^{2}$. If we denote $\left\{\mathbb{V}_{j}, j \in \mathbb{Z}\right\}$ the multiresolution analysis of $\left(L_{\mathrm{p}}^{2}(Y)\right)^{2}$, an element of $\mathbb{V}_{j}$ is thus written:

$$
\mathbf{u}\left(x_{1}, x_{2}\right)=\left(\begin{array}{l}
u_{1}\left(x_{1}, x_{2}\right) \\
u_{2}\left(x_{1}, x_{2}\right)
\end{array}\right)
$$

with

$$
u_{i}\left(x_{1}, x_{2}\right)=\sum_{\left(k_{1}, k_{2}\right) \in \Lambda_{i}} u_{i}^{k_{1} k_{2}} \Phi_{k_{1} k_{2}}^{j}\left(x_{1}, x_{2}\right)
$$

with $N^{j}=2^{i}-1$ and $\Lambda_{j}=\left[0, N^{j}\right]^{2}$.

For simplicity, we have merely chosen to perform the representation on one given level $j$. Note that the dimension of the space $V_{j}^{\mathrm{p}}$ is equal to $2^{j}$ and so the dimension of a space $V_{j}$ is equal to $2^{2 j+1}$.

\section{ELASTOSTATICS DISCRETIZATION}

\subsection{General form}

The operator which we represent on $Y$ is $\operatorname{div} C$ $\operatorname{grad}_{\mathrm{s}}$. A variational formulation, using Green's formulae, leads to the term $\int_{Y} \sigma(\mathbf{u}): \epsilon(\mathbf{v}) \mathrm{d} y . \epsilon$ is the second order strain tensor, $\epsilon=\operatorname{grad}_{s} \mathbf{u} . \sigma$ is the second order Cauchy stress tensor, $\sigma=\mathbf{C} \boldsymbol{\epsilon}$. Let $u_{i, j}=\partial u_{i} / \partial x_{j}$ for $i, j=1,2$. The projection of $\mathbf{u}$ and $\mathbf{v}$ in wavelets' bases corresponds to the representation of the plane elastostatics operators in compactly supported bases. In this case, Proposition 1 is obtained.

Proposition 1: the projection of the plane elasticity operators into the wavelet basis with scale equal to $j$, is given by a matrix $\mathbf{K}$ of order $2^{2 j+1}: \mathbf{K}=\left(K_{(i 1, i 2),(k 1, k 2)}\right)$, where $\left.K_{(i 1, i 2),(k 1, k 2)}\right)=\mathbf{K}_{\mathrm{ik}}$ is an elementary matrix of order 2:

$$
\mathbf{K}_{\mathrm{ik}}=\left[\begin{array}{ll}
F_{11}^{11}+F_{33}^{22} & F_{12}^{12}+F_{33}^{21} \\
F_{21}^{21}+F_{33}^{12} & F_{22}^{22}+F_{33}^{11}
\end{array}\right]
$$

where

$$
\begin{aligned}
F_{\mathrm{A}}^{I}=F_{\mathrm{Aik}}^{I}=\int_{Y} & C_{\alpha \beta}\left(x_{1}, x_{2}\right) \Phi_{i, \eta}^{j} \\
& \times\left(x_{1}, x_{2}\right) \Phi_{k, \xi}^{j}\left(x_{1}, x_{2}\right) \mathrm{d} x_{1} \mathrm{~d} x_{2},
\end{aligned}
$$

with $\mathbf{A}=(\alpha, \beta), \alpha, \beta=1,2$ or 3 and $\Pi=(\eta, \xi)$, $\eta, \xi=1$ or 2 .

Proof: to verify this proposition, we need to define the plane strain tensor. For bi-dimensional problems, the strain tensor $\epsilon$ is defined by

$$
\epsilon_{11}=u_{1,1}, \epsilon_{22}=u_{2.2} \text { and } \epsilon_{12}=1 / 2\left(u_{1.2}+u_{2.1}\right) \text {, }
$$

and the symmetric elasticity tensor $\mathbf{C}=\left(C_{i j}\right)$ by $C_{i 3}=0$ for $i \leqslant 3$. In order to discretize the problem, we need to express the term $\sigma(\mathbf{u}): \epsilon(\mathbf{v})$ :

$$
\begin{aligned}
\boldsymbol{\sigma}(\mathbf{u}): \boldsymbol{\epsilon}(\mathbf{v})= & (\mathbf{C} \boldsymbol{\epsilon}(\mathbf{u})): \boldsymbol{\epsilon}(\mathbf{v})=\left(C_{11} u_{1,1}+C_{12} u_{2,2}\right) v_{1,1} \\
& +\left(C_{12} u_{1,1}+C_{22} u_{2,2}\right) v_{2,2} \\
& +C_{33}\left(u_{1,2}+u_{2,1}\right)\left(v_{1,2}+v_{2,1}\right)
\end{aligned}
$$

This expression is discretized in the wavelet basis with $\mathbf{u}$ given by eqns $(28,29)$ and with $v$ equal to the vectors of the wavelet basis. It follows directly that

$$
\begin{gathered}
\boldsymbol{\sigma}(\mathbf{u}): \boldsymbol{\epsilon}(\mathbf{v})=\sum_{i, k \in \Lambda_{j}}\left(\left(C_{11} u_{1}^{i} \Phi_{i, 1}^{j}+C_{12} u_{2}^{k} \Phi_{k, 2}^{j}\right) \Phi_{\mathrm{p}, 1}^{j}\right) \\
\left.+C_{33}\left(u_{1}^{i} \Phi_{i, 2}^{j}+u_{2}^{k} \Phi_{k, 1}^{j}\right) \Phi_{p, 2}^{j}\right), \quad \forall \mathbf{p} \in \Lambda_{j}
\end{gathered}
$$

if

$$
\mathbf{v}=\left(\begin{array}{c}
\Phi_{\mathrm{p}}^{j} \\
0
\end{array}\right)
$$

and

$$
\begin{gathered}
\boldsymbol{\sigma}(\mathbf{u}): \boldsymbol{\epsilon}(\mathbf{v})=\sum_{i, k \in A_{j}}\left(\left(C_{12} u_{1}^{i} \Phi_{i, 1}^{j}+C_{22} u_{2}^{k} \Phi_{k, 2}^{j}\right) \Phi_{\mathrm{p}, 2}^{j}\right) \\
\left.+C_{33}\left(u_{1}^{i} \Phi_{i, 2}^{j}+u_{2}^{k} \Phi_{k, 1}^{j}\right) \Phi_{p, 1}^{j}\right), \quad \forall \mathbf{p} \in A_{j},
\end{gathered}
$$

if

$$
\mathbf{v}=\left(\begin{array}{c}
0 \\
\Phi_{\mathrm{p}}^{j}
\end{array}\right)
$$

By integration on $Y$, the proof of Proposition 1 is obtained. Q.E.D. It is clear, from the form of the elementary matrix, that the matrix $\mathbf{K}$ is symmetric.

\subsection{Matrix coefficients}

In this section, we propose a technique to compute the terms of the matrix $\mathbf{K}$. $\mathbf{C}$ is usually a piecewise constant function (a given constant for each material). Two cases appear. $\mathbf{C}$ is either a constant function on the intersection of the supports of $\Phi_{i}^{j}$ and $\Phi_{k}^{j}$ or not.

The first case is more easily tractable. Equation (31) gives us 


$$
\begin{aligned}
F_{A}^{n} & =C_{\alpha \beta} \int_{Y} \Phi_{i, \eta}^{j}\left(x_{1}, x_{2}\right) \Phi_{k, \xi}^{j}\left(x_{1}, x_{2}\right) \mathrm{d} x_{1} \mathrm{~d} x_{2}, \\
& =C_{x \beta} A_{i k} B_{i k},
\end{aligned}
$$

where

$$
\left\{\begin{array}{c}
\text { if } \eta=1 \text { then } n 1=1 \text { and } n 2=0, \\
\text { if } \eta=2 \text { then } n 1=0 \text { and } n 2=1, \\
\text { if } \xi=1 \text { then } m 1=1 \text { and } m 2=0, \\
\text { if } \xi=2 \text { then } m 1=0 \text { and } m 2=1, \\
A_{i k}=\int_{0}^{1} \phi_{j i 1}^{(n)}\left(x_{1}\right) \phi_{j k 1}^{(m 1)}\left(x_{1}\right) \mathrm{d} x_{1}, \\
B_{i k}=\int_{0}^{1} \phi_{j i 2}^{(n 2)}\left(x_{2}\right) \phi_{j k 2}^{(m 2)}\left(x_{2}\right) \mathrm{d} x_{2},
\end{array}\right.
$$

and $f^{(s)}$ is the derivative of order $s$.

We note that eqn (38) is a translation of the derivation with respect to the first or second variable. The algorithm to compute the terms $A_{i k}$ and $B_{i k}$ is given in Section 3.3.

On the other hand, if $\mathbf{C}$ is not constant on the intersection of the supports of the two wavelets, this function is decomposed into a wavelet basis which is not necessarily the same as in Section 2.3. Due to the form of $C_{\alpha \beta}$, the best choice seems to be the Haar basis.

Let $\theta$ be the wavelet basis of decomposition of $C_{z \beta}$, $\theta$ is a wavelet basis of $L_{\mathrm{p}}^{2}(0,1)$. We obtain the following formula:

$$
C_{x \beta}\left(x_{1}, x_{2}\right)=\sum_{\left(\rho_{1}, \rho_{2}\right) \in A_{j}} C_{\alpha \beta}^{\rho_{1}, \rho_{2}} \theta_{J_{j, 1}}\left(x_{1}\right) \theta_{J_{p_{2}}}\left(x_{2}\right)
$$

with $N^{J}=2^{J}-1$ and $A_{J}=\left[0, N^{J}\right]^{2}$.

$J$ is not necessarily equal to $j$, but usually $J \geqslant j$ is chosen to have $2^{s-j}$ in $\mathbb{N}$.

Proposition 2: the evaluation of the terms $F_{\mathrm{A}}^{I}$ needs to compute the elementary terms $\Gamma_{J j i k}^{\mathbf{P}}, \mathbf{P}=(n, m)$, where

$$
\Gamma_{J j i k}^{\mathbf{P}}=\int_{0}^{1} \theta_{J k}(x) \phi_{j i}^{(n)}(x) \phi_{j 0}^{(m)}(x) \mathrm{d} x
$$

More precisely,

$$
F_{\mathrm{A}}^{n}=\sum_{\left(\rho_{1}, \rho_{2}\right) \in \Lambda_{j}} C_{A}^{\rho_{1}, \rho_{2}} \Gamma_{J f r_{1} s_{1}}^{\mathrm{P}_{1}} \Gamma_{J j r_{2} s_{2}}^{\mathrm{P}_{2}}
$$

with $r_{x}=i_{x}-k_{x}$ and $s_{x}=\rho_{x}-2^{J-i} k_{z}$. The same notations are used as in eqn (31).

Proof: using eqn (41), the different terms $F_{A}^{h}$ of the matrix $\mathbf{K}$ have the following form:

$$
F_{A}^{n}=\sum_{\rho \in A_{j}} C_{A}^{\rho} A_{i k \rho} B_{i k \rho}
$$

with:

$$
\begin{gathered}
\rho=\left(\rho_{1}, \rho_{2}\right) \\
A_{i k \rho}=\int_{0}^{1} \theta_{J_{\rho 1}}\left(x_{1}\right) \phi_{j i !}^{(m 1)}\left(x_{1}\right) \phi_{j k 1}^{(m !)}\left(x_{1}\right) \mathrm{d} x_{1},
\end{gathered}
$$

and

$$
B_{i k \rho}=\int_{0}^{1} \theta_{J_{\rho_{2}}}\left(x_{2}\right) \phi_{j i 2}^{(n 2)}\left(x_{2}\right) \phi_{j k 2}^{(m 2)}\left(x_{2}\right) \mathrm{d} x_{2} .
$$

Using a simple change of variables, we obtain

$$
\begin{aligned}
\int_{0}^{1} \theta_{J_{\mathrm{p}}}(x) \phi_{j i}^{(n)}(x) & \phi_{j k}^{(m)}(x) \mathrm{d} x \\
& =\int_{0}^{1} \theta_{J_{\mathrm{r}}}(x) \phi_{j . s}^{(n)}(x) \phi_{j 0}^{(m)}(x) \mathrm{d} x
\end{aligned}
$$

with $r=i-2^{J-j} k$ and $s=k-i$. Q.E.D.

Thus, we have to compute elementary terms which can be seen as the "product of three wavelets". The algorithm to compute these terms is given in Section 3.4.

\subsection{Evaluation of two wavelets' products}

The evaluation of the integral $\int_{0}^{1} \phi_{j i}^{(n)}(x) \phi_{j k}^{(m)}(x) \mathrm{d} x$ is a particular case of a theorem given by Beylkin [3].

Theorem 1: let

$$
r_{k}^{n}=\int_{\mathbb{R}} \varphi(x-k) \varphi^{(n)}(x) \mathrm{d} x, n \in \mathbb{N}
$$

Then,

$$
r_{k}^{n}=2^{n}\left[r_{2 k}^{n}+\frac{1}{2} \sum_{m=1}^{M} a_{2 m-1}\left(r_{2 k-2 m+1}^{n}+r_{2 k+2 m-1}^{n}\right)\right]
$$

and

$$
\sum_{k} k^{n} r_{k}^{n}=(-1)^{n} n !
$$

with

$$
a_{k}=2 \sum_{i=0}^{2 M-1-k} h(i) h(i+k), k=1,2 M-1 .
$$

The coefficients $h(i)$ are defined in eqns $(5,6)$. This theorem corresponds to the evaluation of 


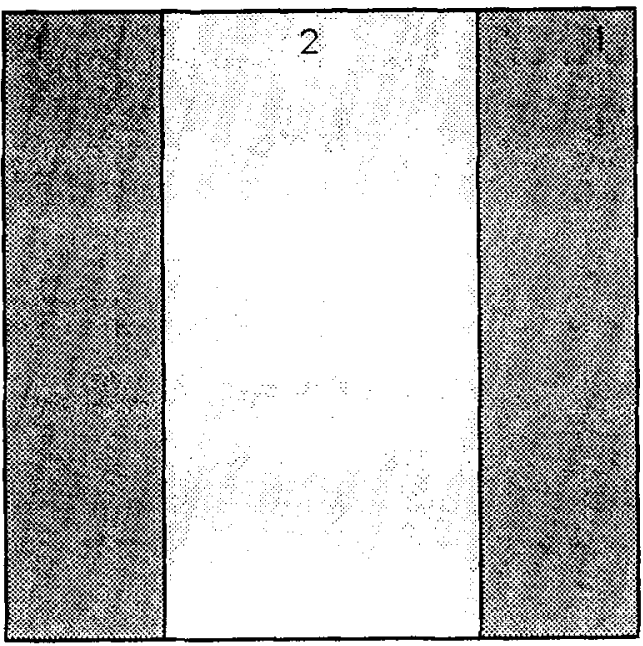

Fig. 4. Elementary composite (1) Young's modulus: $410000 \mathrm{MPa}$, Poisson's ratio: 0.19. (2) Young's modulus: $72000 \mathrm{MPa}$, Poisson's ratio: 0.32 .

tors of a simple matrix with eigenvalues equal to $2^{-n}$. Usually, due to the low dimension of the matrix, this evaluation can be done by a symbolic computational software. Using eqn (24), the evaluation of the terms $\int_{0}^{1} \phi_{j i}^{(1)}(x) \phi_{j k}^{(0)}(x) \mathrm{d} x$ is given by the theorem with $n=1$ and the evaluation of the terms $\int_{0}^{1} \phi_{j i}^{(1)}(x) \phi_{j k}^{(1)}(x) \mathrm{d} x$ by the theorem with $n=2$.

\subsection{Evaluation of the three wavelets' products}

To perform the terms $\Gamma_{J_{j i k}}^{\mathrm{P}}$ it is necessary to introduce the coefficients $\gamma_{i k}^{\mathbf{P}}$ where

$$
\gamma_{i k}^{\mathbf{P}}=\int_{\mathbb{B}} \theta(x) \varphi^{(n)}(x-i) \varphi^{(m)}(x-k) \mathrm{d} x,
$$

where $\theta$ is a Daubechies wavelet of $\operatorname{order} \mathbb{M}$ and $\varphi$ a Daubechies wavelet of order $M$. These wavelets are chosen without periodic conditions.

The two wavelets verify the following conditions:

$$
\begin{array}{ll}
\theta(x)=\sqrt{2} \sum_{r=0}^{r-2 \mathbb{A}-1} H(r) \theta(2 x-r), & \forall x \in \mathbb{R}, \\
\varphi(x)=\sqrt{2} \sum_{r=0}^{r=2 M-1} h(r) \varphi(2 x-r), \quad \forall x \in \mathbb{R} .
\end{array}
$$

The following theorem is introduced.

Theorem 2: the coefficients $\gamma_{i k}^{\mathrm{P}}$, verify the relation

$$
\gamma_{i k}^{\mathbf{p}}=2^{n+m} \sum_{\alpha, \beta=0}^{2 M-1} \mathbb{E}_{i k \alpha \beta} \gamma_{\alpha \beta}^{\mathbf{p}},
$$

with

$$
\mathbb{B}_{i k \alpha \beta}=\sqrt{8} \sum_{r=0}^{2 M-1} H(r) h
$$

$$
\begin{aligned}
& \times(\alpha-2 i+r) h(\beta-2 k+r) \chi_{[0,2 M-1]} \\
& \times(\alpha-2 i+r) \chi_{[0,2 M-1]}(\beta-2 k+r),
\end{aligned}
$$

and

$$
\begin{aligned}
& \chi_{[a, b]}(x)=1 \text { if } x \in[a, b], \\
& \chi_{[a, b]}(x)=0 \text { in all other cases. }
\end{aligned}
$$

Proof: due to eqns $(53,54)$,

$$
\gamma_{i k}^{\mathbf{P}}=2^{n+m} \sqrt{2} \sum_{\substack{r=0 \\ r i, r 2=0}}^{\substack{r=2 \mathrm{M}-1 \\ r 1, r 2=2 M-1}} H(r) h(r 1) h(r 2) \gamma_{q s}^{\mathbf{P}},
$$

with $q=2 i+r 1-r$ and $s=2 k+r 2-r$. The change of variables $i 1=2 i+r 1-r$ and $k 1=2 k+r 2-r$ and the equality $\gamma_{i k}^{\mathrm{P}}=0$ for $i, k \notin[2-2 M, 2 M-2]$, complete the proof. Q.E.D.

$\mathbb{B}$ is a fourth order tensor. The coefficients $\gamma$ can then be seen as eigenmatrices of the tensor $\mathbb{B}$. The evaluation of these terms is obtained using a wellchosen numbering. The tensor $\mathbb{B}$ is transformed into a matrix and the matrix $\gamma$ into a vector. Then, eqn (55) is a problem of the determination of eigenvectors with the eigenvalue $2^{n+m}$. Note that this problem is solved only once and written in filter banks.

Details and tables concerning the computations of the coefficients are given in the Appendix 1 .

The evaluation of the coefficients $\Gamma$ is obtained using the following formula, obtained using a simple change of variables in eqn (52) and for $J$ sufficiently larger than 0 :

$$
\Gamma_{J J i k}^{\mathbf{P}}=2^{(n+m+3 / 2) \gamma_{\gamma}} \gamma_{(k-i)(-i)}^{\mathbf{P}} .
$$

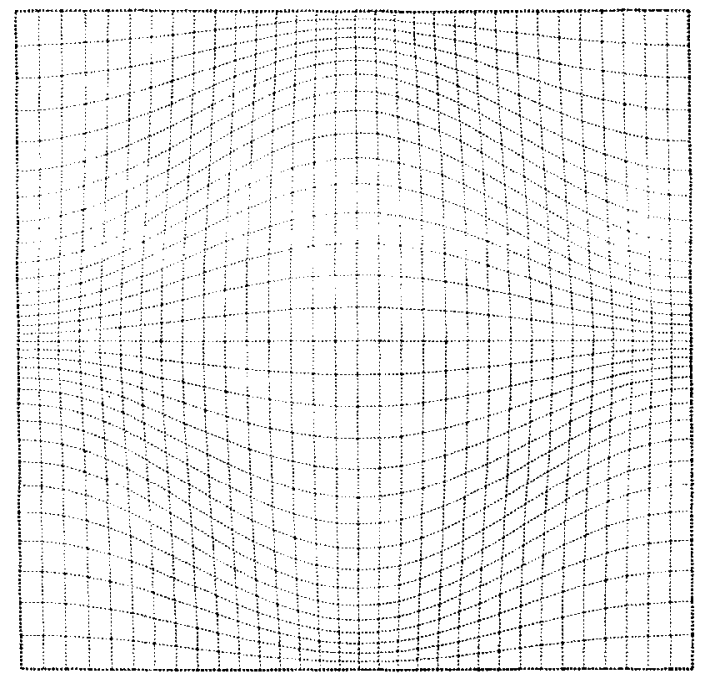

Fig. 5. Displacement $\mathbf{u}\left(x_{1}, x_{2}\right), M=3, j=5$. 


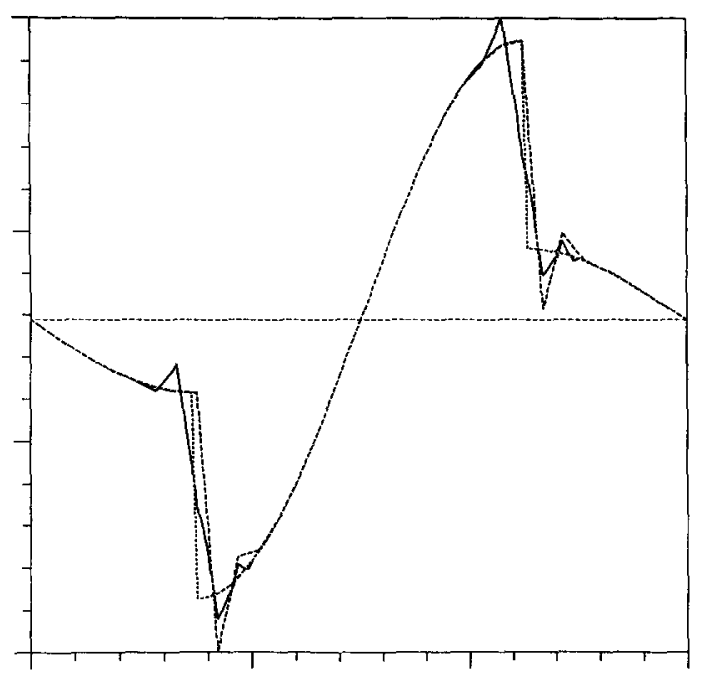

Fig. 6. First component of the force $f_{1}\left(x_{1}, 0.5\right)$ (analytical results, numerical results and direct decomposition).

Then, the coefficients $\Gamma_{J i k}^{\mathrm{P}}(j<J)$ are performed from eqn (59) by a pyramidal algorithm described for example in Refs [2,6 and 7] (see Appendix 2).

\section{VALIDATION TEST}

The aim of this paragraph is to validate the representation from an academic example. We consider an elementary composite (see Fig. 4). Let the displacement $u$ be

$$
\begin{gathered}
u_{1}\left(x_{1}, x_{2}\right)=\sin \left(2 \pi x_{1}\right) \cos \left(2 \pi x_{2}\right), \\
u_{2}\left(x_{1}, x_{2}\right)=10 \cos \left(2 \pi x_{1}\right) \sin \left(2 \pi x_{2}\right) .
\end{gathered}
$$

The forces applied on the domain are given on each sub-domain by the following formulae:

$$
\begin{aligned}
& f_{1}\left(x_{1}, x_{2}\right)=4 \pi^{2}\left(C_{11}+10 C_{12}\right. \\
&\left.+11 C_{33}\right) \sin \left(2 \pi x_{1}\right) \cos \left(2 \pi x_{2}\right) \\
& f_{2}\left(x_{1}, x_{2}\right)=4 \pi^{2}\left(C_{12}+10 C_{22}\right. \\
&\left.+11 C_{33}\right) \cos \left(2 \pi x_{1}\right) \sin \left(2 \pi x_{2}\right)
\end{aligned}
$$

The displacement given by eqns $(60,61)$ is projected into the wavelet basis $(M=3)$ and transformed by the representation of the plane strain elastostatics operator. The forces obtained are compared with the analytical solution given by eqns $(62,63)$ and with the direct decomposition of this solution into the wavelet basis. Figures 5 and 6 show the accuracy between the numerical and analytical results. Figure 5 represents the displacement $\mathbf{u}=\left(u_{1}, u_{2}\right)$ and Fig. 6, the force $f_{1}\left(x_{1}, x_{2}\right)$ for $x_{2}=0.5$.

\section{CONCLUSION}

The numerical model presented in this paper turns out to be a good alternative to the finite element methods and Fourier analysis. Several applications can be made: periodic homogenization [8], linear elasticity using fictitious domains [9], inverse problems using experimental data, linear and non-linear properties of a composite from images of its complex microstructure, etc. On the other hand, the first improvements to give are the use of adaptivity properties and the implementation of wavelets with Dirichlet and Neumann boundary conditions.

\section{REFERENCES}

1. I. Daubechies, Orthonormal bases of compactly supported wavelets. Comm. pure appl. Math. 41, 909--996 (1988).

2. G. Beylkin, R. Coifman and V. Rokhlin, Fast wavelet transforms and numerical algorithms I. CPAMS 44, 141-183 (1991).

3. G. Beylkin and B. Torrésani, Implementation of operators via filter banks, autocorrelation shell and Hardy wavelets. Preprint CPT-94/P.3009 (1994).

4. G. Beylkin and B. Torrésani, On the representation of operators in bases of compactly supported wavelets SIAM J. numer. Anal. 6, 1716-1760 (1992).

5. J. Liandrat, V. Perrier and $P$. Tehamitchian, Numerical resolution of non-linear partial differential equations using the wavelet approach. In: Wavelets and their applications, pp. 227-238. Jones and Barlet (1992).

6. S. Dumont and F. Lebon, Ondelettes et équations aux dérivées partielles. I. Le cas unidimensionnel. Note Interne no. 93-1, Laboratoire de Mécanique et Génie Civil, Université Montpellier 2 (1993).

7. A. Cohen, Ondelettes et traitement numérique du signal, RMA 25, Masson, Paris (1992).

8. S. Dumont and F. Lebon, Wavelet-Galerkin method for periodic heterogeneous media. (1996) to appear in Computers \& Structures (submitted).

Table A1. Coefficients $\gamma_{i k}^{00}(M=1$ and $M=3)$

\begin{tabular}{lrr}
\hline$i$ & $k$ & $\gamma$ \\
\hline 0 & -4 & $-0.441328283 \times 10^{-3}$ \\
0 & -3 & $-0.187931357 \times 10^{-1}$ \\
0 & -2 & $0.123328235 \times 10^{0}$ \\
0 & -1 & $0.496647798 \times 10^{0}$ \\
0 & 0 & 0 \\
1 & -3 & $0.441328283 \times 10^{-3}$ \\
1 & -2 & $0.189275244 \times 10^{-1}$ \\
1 & -1 & $-0.117675941 \times 10^{0}$ \\
1 & 0 & $0.470948730 \times 10^{11}$ \\
1 & -1 & $0.123328235 \times 10^{4}$ \\
2 & -2 & $-0.134388718 \times 10^{-3}$ \\
2 & -1 & $-0.568478777 \times 10^{-2}$ \\
2 & 0 & $0.310635327 \times 10^{-1}$ \\
2 & 1 & $-0.117675941 \times 10^{0}$ \\
2 & 2 & $-0.187931357 \times 10^{-1}$ \\
3 & -1 & $0.324935170 \times 10^{-4}$ \\
3 & 0 & $0.133846091 \times 10^{-2}$ \\
3 & 1 & $-0.568478777 \times 10^{-2}$ \\
3 & 2 & $0.189275244 \times 10^{-1}$ \\
3 & 3 & $-0.441328283 \times 10^{-3}$ \\
4 & 0 & $0.147791132 \times 10^{-5}$ \\
4 & 1 & $0.324935170 \times 10^{-4}$ \\
4 & 2 & $-0.134388718 \times 10^{-3}$ \\
4 & 3 & $0.441328283 \times 10^{-3}$ \\
4 & 4 & 0 \\
\hline & & \\
\hline
\end{tabular}


9. S. Dumont, F. Lebon and P. Alart, Elasticité et ondelettes: formulations mathématiques et mise en oeuvre numérique. In: Proc. 2nd French National Congress on Computational Structural Mechanics, Giens, France pp. 827-832 Hermes, Paris (1995) (in press).

\section{APPENDIX 1}

\section{Computation of $\gamma_{i k}^{0 n}$}

To perform the coefficients $\gamma_{i k}^{00}$ it is necessary to determine an eigenvector of the matrix $\mathbb{B}$ with the eigenvalue equal to one. Another condition has to be introduced to satisfy the uniqueness of this vector.

The functions $\theta$ and $\varphi$ verify:

$$
\begin{gathered}
\sum_{k \in \mathbb{Z}} \theta(x-k)=1, \quad \forall x \in \mathbb{R}, \\
\int_{\mathbb{R}} \varphi(x) \varphi(x-k) \mathrm{d} x=\delta_{0 k}, \quad \forall k \in \mathbb{Z} .
\end{gathered}
$$

Then

$$
\sum_{k \in \mathbb{Z}} \gamma_{k k}^{\infty 0}=1
$$

Table Al gives the values of the vector $\gamma_{i k}^{00}$,

\section{Computation of $\gamma_{i k}^{01}$}

The coefficients $\gamma_{i k}^{01}$ correspond to the determination of an eigenvector of the matrix $\mathbb{B}$ with the eigenvalue equal to 0.5 . Let

$$
\delta_{i k}=\int_{\mathbb{R}} \theta^{(l)}(x) \varphi(x-i) \varphi(x-k) \mathrm{d} x .
$$

Then, $\gamma_{i k}^{01}$ and $\delta_{i k}$ are two non-colinear eigenvectors of $\mathbb{B}$. Due to the definition of $\gamma_{i k}^{01}$ and $\delta_{i k}$, then

Table A2. Coefficients $\gamma_{i k}^{01}(M=I$ and $M=3)$

\begin{tabular}{lrc}
\hline$i$ & $k$ & $\gamma$ \\
\hline 0 & -4 & $0.342465753 \times 10^{-3}$ \\
0 & -3 & $0.150539701 \times 10^{-1}$ \\
0 & -2 & $-0.124283163 \times 10^{0}$ \\
0 & -1 & $0.567892841 \times 10^{0}$ \\
0 & 0 & $0.827328955 \times 10^{0}$ \\
1 & -3 & $-0.442097978 \times 10^{-3}$ \\
1 & -2 & $-0.211110611 \times 10^{-1}$ \\
1 & -1 & $0.166483459 \times 10^{0}$ \\
1 & 0 & $-0.752893875 \times 10^{0}$ \\
1 & 1 & $-0.106420845 \times 10^{1}$ \\
2 & -2 & $0.188744849 \times 10^{-3}$ \\
2 & -1 & $0.109093267 \times 10^{-1}$ \\
2 & 0 & $-0.698971276 \times 10^{-1}$ \\
2 & 1 & $0.293074414 \times 10^{0}$ \\
2 & 2 & $0.246829148 \times 10^{0}$ \\
3 & -1 & $-0.801477819 \times 10^{-4}$ \\
3 & 0 & $-0.452898781 \times 10^{-2}$ \\
3 & 1 & $0.262518093 \times 10^{-1}$ \\
3 & 2 & $-0.103068691 \times 10^{0}$ \\
3 & 3 & $-0.960718286 \times 10^{-2}$ \\
4 & 0 & $-0.896484317 \times 10^{-5}$ \\
4 & 1 & $-0.323247933 \times 10^{-3}$ \\
4 & 2 & $0.144502219 \times 10^{-2}$ \\
4 & 3 & $-0.500468928 \times 10^{-2}$ \\
4 & 4 & $-0.342465753 \times 10^{-3}$ \\
\hline
\end{tabular}

Table A3. Coefficients $\gamma_{i k}^{\prime 1}(M=1$ and $M=3)$

\begin{tabular}{lrr}
\hline$i$ & $k$ & $\gamma$ \\
\hline 0 & -4 & $-0.535714856 \times 10^{-2}$ \\
0 & -3 & $-0.122693167 \times 10^{0}$ \\
0 & -2 & $0.677531606 \times 10^{0}$ \\
0 & -1 & $-0.225189020 \times 10^{1}$ \\
0 & 0 & $0.170240891 \times 10^{1}$ \\
1 & -3 & $0.840745335 \times 10^{-2}$ \\
1 & -2 & $0.203438334 \times 10^{0}$ \\
1 & -1 & $-0.101181720 \times 10^{1}$ \\
1 & 0 & $0.305186162 \times 10^{1}$ \\
1 & 1 & $-0.225189020 \times 10^{1}$ \\
2 & -2 & $-0.477946449 \times 10^{-2}$ \\
2 & -1 & $-0.128299571 \times 10^{0}$ \\
2 & 0 & $0.467364635 \times 10^{0}$ \\
2 & 1 & $-0.101181720 \times 10^{1}$ \\
2 & 2 & $0.677531606 \times 10^{0}$ \\
3 & -1 & $0.153079476 \times 10^{-2}$ \\
3 & 0 & $0.460236098 \times 10^{-1}$ \\
3 & 1 & $-0.128299571 \times 10^{0}$ \\
3 & 2 & $0.203438334 \times 10^{0}$ \\
3 & 3 & $-0.122693167 \times 10^{0}$ \\
4 & 0 & $0.198359243 \times 10^{-3}$ \\
4 & 1 & $0.153079476 \times 10^{-2}$ \\
4 & 2 & $-0.477946449 \times 10^{-2}$ \\
4 & 3 & $0.840745335 \times 10^{-2}$ \\
4 & 4 & $-0.535714856 \times 10^{-2}$ \\
\hline
\end{tabular}

$$
\begin{gathered}
\sum_{\substack{p \in \mathbb{Z} \\
k=2-2 M}}^{k=2 M-2} k \gamma_{-p k-p}^{01}=1, \quad \sum_{\substack{p \in \mathbb{Z} \\
k=2-2 M}}^{k=2 M-2} k \delta_{-p k-p}=0, \\
\sum_{p \in \mathbb{Z}} \gamma_{k p}^{01}=0, \quad \forall k \in \mathbb{Z} \quad \text { and } \sum_{\substack{p \in \mathbb{Z} \\
k=2-2 M}}^{k=2 M-2} k \gamma_{k p}^{01}=1 .
\end{gathered}
$$

So, the technique to perform the coefficients consists of obtaining two non-colinear eigenvectors $\mathbf{A}$ and $\mathbf{B}$, and to determine $\alpha$ and $\beta$, such that:

$$
\begin{gathered}
\alpha \sum_{\substack{p \in \mathbb{Z} \\
k=2-2 M}}^{k=2 M-2} k A_{k p}+\beta \sum_{\substack{p \in \mathbb{Z} \\
k=2-2 M}}^{k=2 M-2} k B_{k p}=0, \\
\alpha \sum_{\substack{p \in \mathbb{Z} \\
k=2-2 M}}^{k=2 M-2} k A_{p k-p}+\beta \sum_{\substack{p \in \mathbb{Z} \\
k=2-2 M}}^{k=2 M-2} k B_{-p k-p}=1 .
\end{gathered}
$$

Therefore,

$$
\gamma=\alpha \mathbf{A}+\beta \mathbf{B} .
$$

Table A2 gives the values of the vector $\gamma_{i k}^{01}$.

\section{Computation of $\gamma_{i k}^{11}$}

The coefficients $\gamma_{i k}^{\prime \prime}$ correspond to the determination of an eigenvector of the matrix $\mathbb{B}$ with the eigenvalue equal to 0.25 . Let

$$
\delta_{i k}=\int_{\mathbb{R}} \theta(x-i) \varphi^{(1)}(x-k) \varphi^{(1)}(x) \mathrm{d} x,
$$

and

$$
\epsilon_{i k}=\int_{\mathrm{R}} \theta(x-i) \varphi(x-k) \varphi^{(2)}(x) \mathrm{d} x
$$




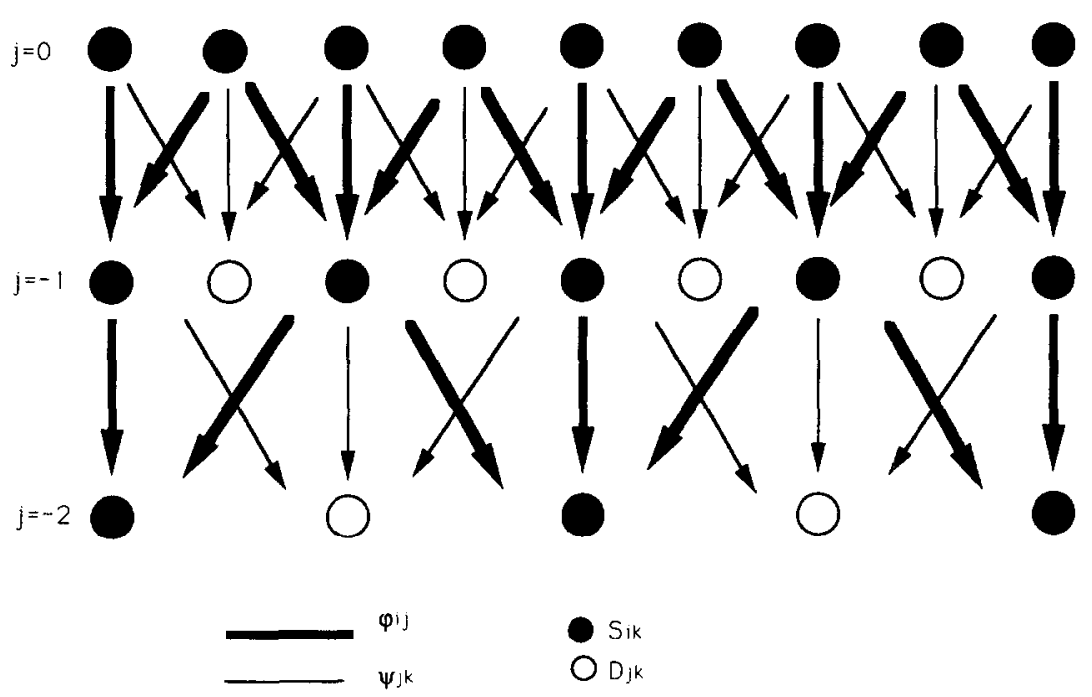

Fig. Al. Fast wavelet transform for $j=-2$.

Then, the vectors $\delta_{i k}, \epsilon_{i k}$ and $\gamma_{i k}^{\prime \prime}$ are linearly dependent and are the three eigenvectors of $\mathbb{B}$ with an eigenvalue 0.25 . To find the vector $\gamma$, a method similar to the method introduced in the former section is used. The condition to impose is

$$
\sum_{\substack{p \in \mathbb{Z} \\ k=2-2 M}}^{k=2 M-2} k^{2} \gamma_{k r}=0
$$

Table A3 gives the values of the vector $\gamma_{i k}^{11}$.

\section{APPENDIX 2}

The one-dimensional pyramidal algorithm

Let $J=0$ and $S_{k}^{0}$ be given, $\quad S_{k}^{0}=\int_{Y} f \varphi_{0 k} \mathrm{~d} y$. and

$$
\begin{aligned}
& \text { Let } S_{k}^{\prime}=2^{j / 2} \int_{Y} f \varphi_{j k} \mathrm{~d} y \\
& D_{k}^{j}=2^{j / 2} \int_{Y} f \psi_{j k} \mathrm{~d} y .
\end{aligned}
$$

One can show that

$$
S_{k}^{j}=\sum_{n \in \mathbb{Z}} h(n-2 k) S_{n}^{j+1}
$$

and

$$
D_{k}^{j}=\sum_{n \in \mathbb{Z}} g(n-2 k) S_{n}^{j+1}
$$

Then the coefficients at level $j$ are obtained from the coefficients at level $j+1$ using eqn (A14). This algorithm is presented on an elementary example, with $j=-2$, on Fig. Al. 